\title{
Alignment Test Results of the JWST Pathfinder Telescope Mirrors in the Cryogenic Environment
}

\author{
Tony L. Whitman ${ }^{\mathrm{a}}$, Conrad Wells ${ }^{\mathrm{a}}$, James Hadaway ${ }^{\mathrm{b}}$, J. Scott Knight ${ }^{\mathrm{c}}$, Sharon Lunt ${ }^{\mathrm{a}}$
}

${ }^{a}$ Harris Corp., Space and Intelligence Systems, 400 Initiative Drive, P.O. Box 60488, Rochester, NY, USA 14606; 'bniversity of Alabama, Huntsville, Al; ${ }^{\mathrm{b} B a l l}$ Aerospace \& Technology Corp, 1600 Commerce St., Boulder, CO, USA 80301

\begin{abstract}
After integration of the Optical Telescope Element (OTE) to the Integrated Science Instrument Module (ISIM) to become the OTIS, the James Webb Space Telescope OTIS is tested at NASA's Johnson Space Center (JSC) in the cryogenic vacuum Chamber A for alignment and optical performance.

The alignment of the mirrors comprises a sequence of steps as follows: The mirrors are coarsely aligned using photogrammetry cameras with reflective targets attached to the sides of the mirrors. Then a multi-wavelength interferometer is aligned to the 18-segment primary mirror using cameras at the center of curvature to align reflected light from the segments and using fiducials at the edge of the primary mirror. Once the interferometer is aligned, the 18 primary mirror segments are then adjusted to optimize wavefront error of the aggregate mirror. This process phases the piston and tilt positions of all the mirror segments. An optical fiber placed at the Cassegrain focus of the telescope then emits light towards the secondary mirror to create a collimated beam emitting from the primary mirror. Portions of the collimated beam are retro-reflected from flat mirrors at the top of the chamber to pass through the telescope to the SI detector. The image on the detector is used for fine alignment of the secondary mirror and a check of the primary mirror alignment using many of the same analysis techniques used in the on-orbit alignment.
\end{abstract}

The entire process was practiced and evaluated in 2015 at cryogenic temperature with the Pathfinder telescope.

Keywords: James Webb Space Telescope, OTIS, optical alignment, photogrammetry, interferometer, multi-wavelength

\section{INTRODUCTION}

The cryogenic vacuum optical test for OTIS requires aligning 18 primary mirror segments and the secondary mirror without the ability to look at a star, or a point source at virtual infinity. A Pathfinder telescope with an ISIM surrogate was recently used in the cryogenic environment to practice this alignment with great success, valuable training, and numerous process improvements ${ }^{1}$. After describing the test configuration as it relates to this alignment process, the alignment will be described along with the results from the Pathfinder test followed by the differences expected in the OTIS test.

\section{PATHFINDER OPTICAL TEST CONFIGURATION}

The Pathfinder telescope contains two primary mirror segment assemblies (PMSAs) similar to the flight mirrors (Figure 1). One PMSA is finely polished for cryogenic temperatures and coated with gold. The other PMSA has a less polished surface and is uncoated Beryllium. The two PMSAs are adjacent on the backplane underneath a 1.5-meter autocollimating flat mirror hanging from the top of the chamber. The secondary mirror is also similar to the flight secondary mirror except without the gold coating. For the tertiary mirror and the fine steering mirror the actual flight Aft Optical Subsystem (AOS) was used in the Pathfinder test before integration into the flight telescope early this year.

Underneath the AOS was a detector similar to the SI detectors on three stages to move the detector along the image surface of the AOS. On top of the AOS were optical point sources, mostly fed by optical fibers, at the Cassegrain focus of the telescope injecting light down through the AOS and sources injecting light up to the Secondary Mirror (Figure 1). 


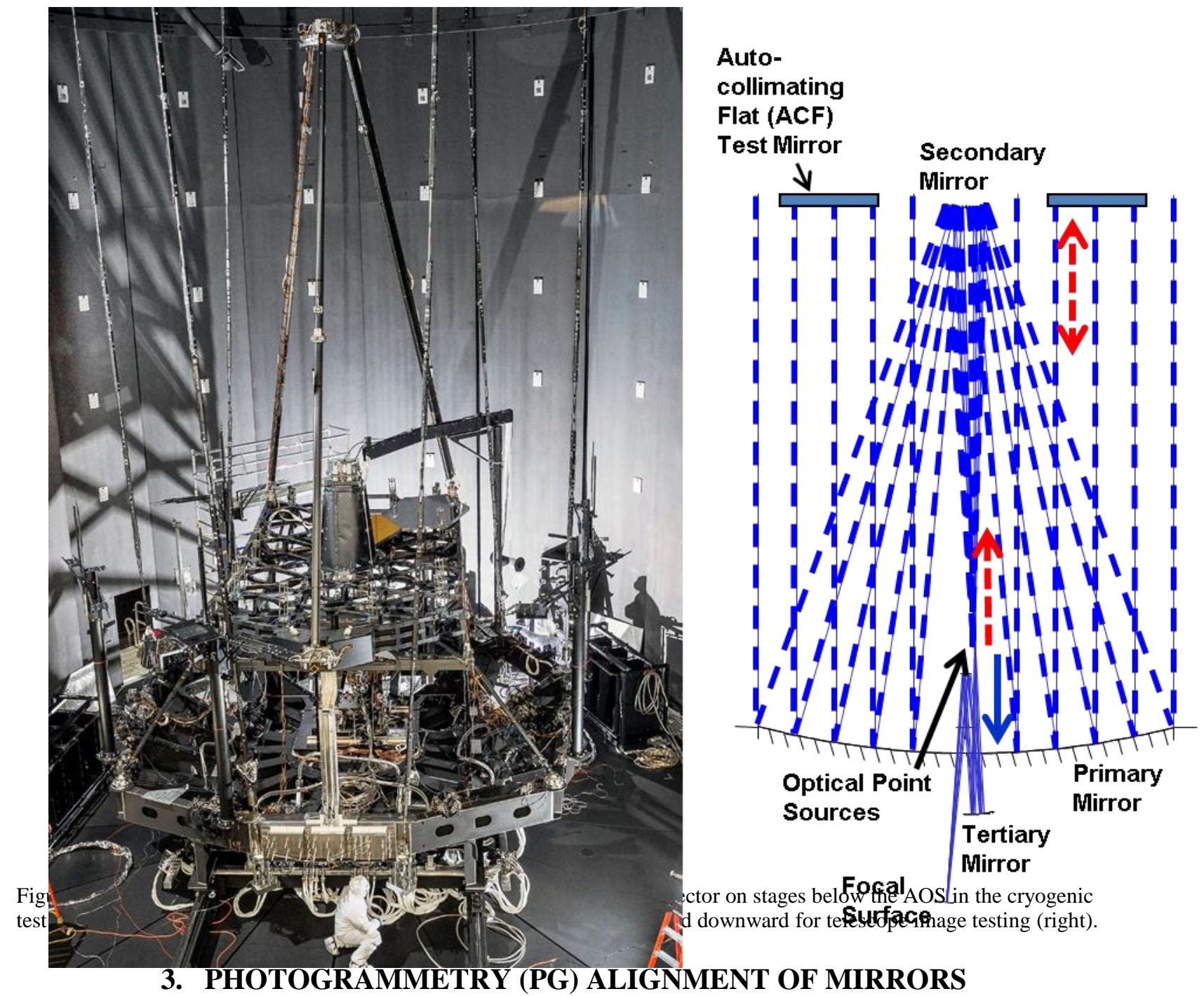

Upon reaching the target test temperature, the first step was aligning the PMSAs and the SMA relative to the AOS. Nine retro-reflective round PG Targets were mounted on the AOS and on the optical source plate assembly (ASPA) (Figure 2). Ten targets were mounted on the PMSAs - one on each hexagon edge except the conjoining edges. And six targets were mounted on the SM. Four cameras in the chamber above the SM rotated on windmill booms to capture the photogrammetry pictures for alignment determination (Figure 3).

Nominal target positions for the mirrors were generated prior to the test in an optical model using prior measurements of the optical surfaces relative to the target interfaces on the mirrors and the AOS, PG target mount design dimensions and measurements, and FEA of gravity sag and cryogenic shift effects. 


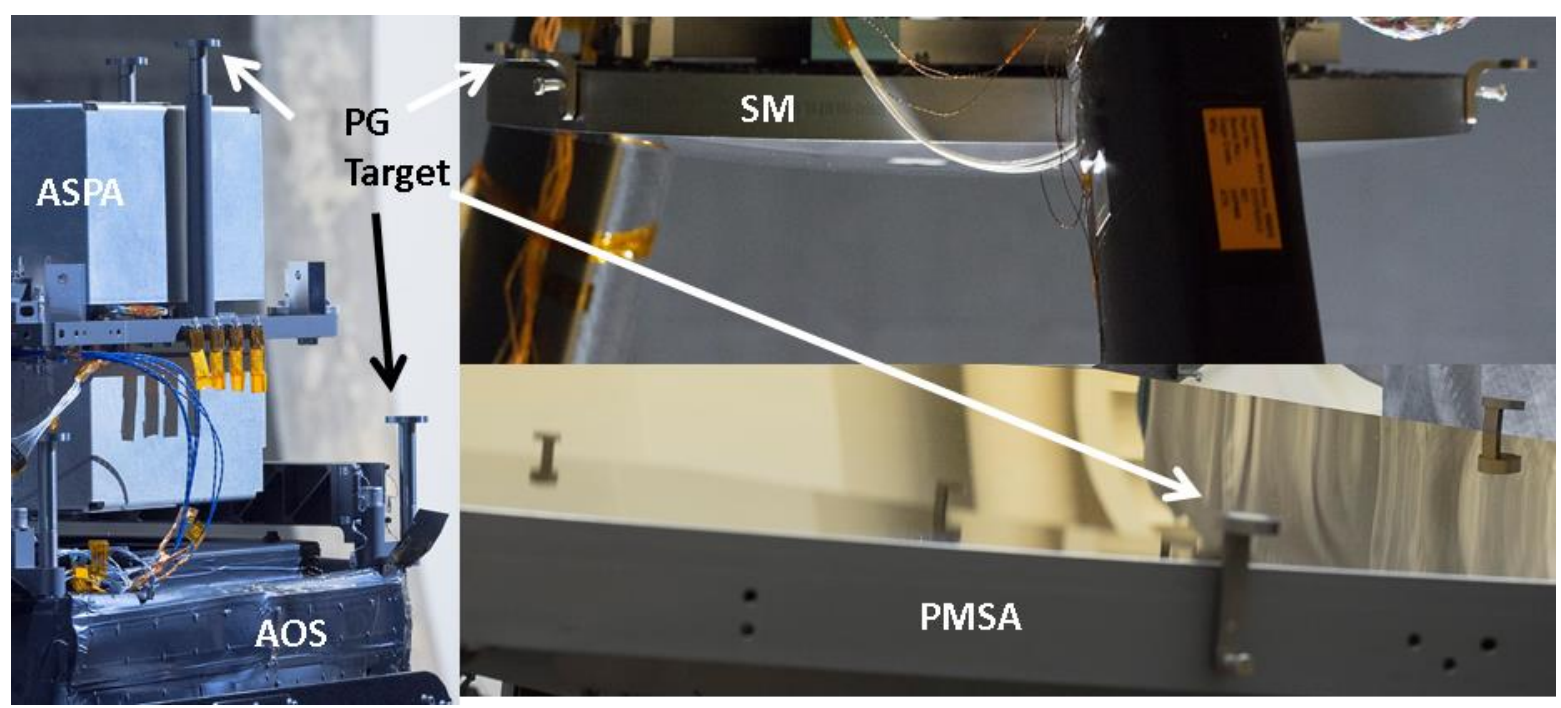

Figure 2 Example PG targets on ASPA, AOS, SM and PMSA

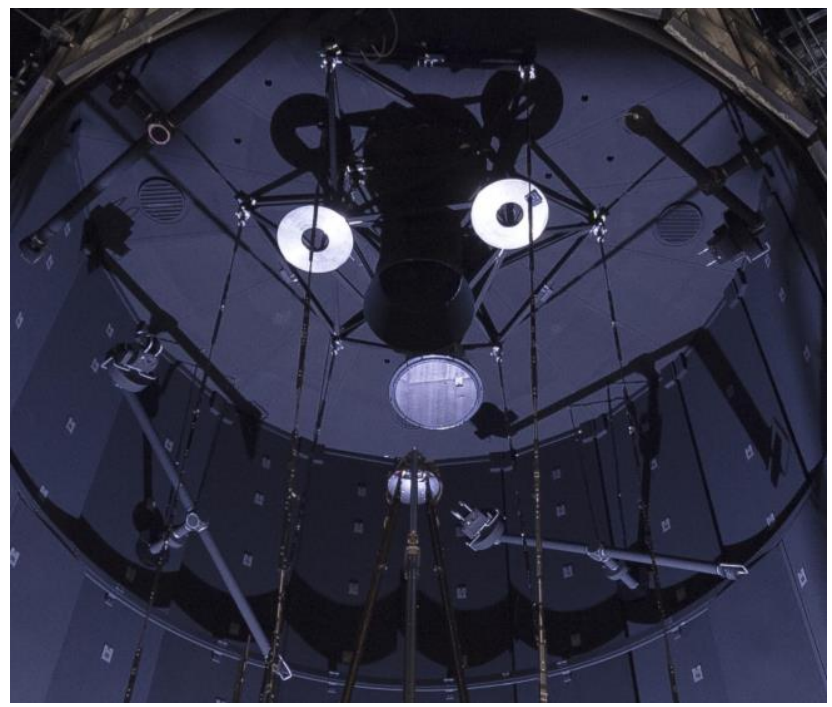

Figure 3 Photogrammetry cameras on windmill booms above the SM and the ACF mirror above the two PMSAs. The COCOA peers through the opening at the center of the ceiling.

Table 1 Photogrammetry metrology uncertainty

\begin{tabular}{|c|c|c|}
\hline \multirow{3}{*}{ PM - AOS } & Degree of freedom & Uncertainty (mm or mrad) \\
\hline \multirow{3}{*}{} & Piston & 0.038 \\
\cline { 2 - 3 } & Decenter & 0.084 \\
\cline { 2 - 3 } & Tilt & 0.090 \\
\cline { 2 - 3 } & Clocking & 0.309 \\
\hline \multirow{3}{*}{ SM - AOS } & Piston & 0.086 \\
\cline { 2 - 3 } & Decenter & 0.652 \\
\cline { 2 - 3 } & Tilt & 0.274 \\
\hline
\end{tabular}


In prior tests, the PG measurements were compared to alternate metrology techniques including laser tracker and laser radar measurements. Around the backplane multiple invar bars with PG targets are placed for scale reference at cryo temperatures. From this prior testing and from repeatability measurements in this test, the measurement uncertainty was determined and is shown in Table 1. This puts the PMSAs to within 100 micrometers of the nominal alignment for most degrees of freedom and places the SM within a millimeter. The SMA uncertainty is larger because small uncertainties in AOS tilt leads to larger centering and tilt uncertainties for the SMA that is seven meters away.

\section{ALIGNING THE INTERFEROMETER TO THE PRIMARY MIRROR}

Then the optical bench with the interferometer and alignment cameras was moved to the average center of curvature location of the PMSAs. The Center of Curvature Optical Assembly (COCOA) ${ }^{3,4,5}$, Figure 4, contains a Coarse Alignment Subsystem (CASS) for finding the interferometer illumination return to the COCOA and steering the return into the field of view of the Fine Alignment Subsystem (FASS) which, in turn, steers the return light into the field of view of the interferometer.

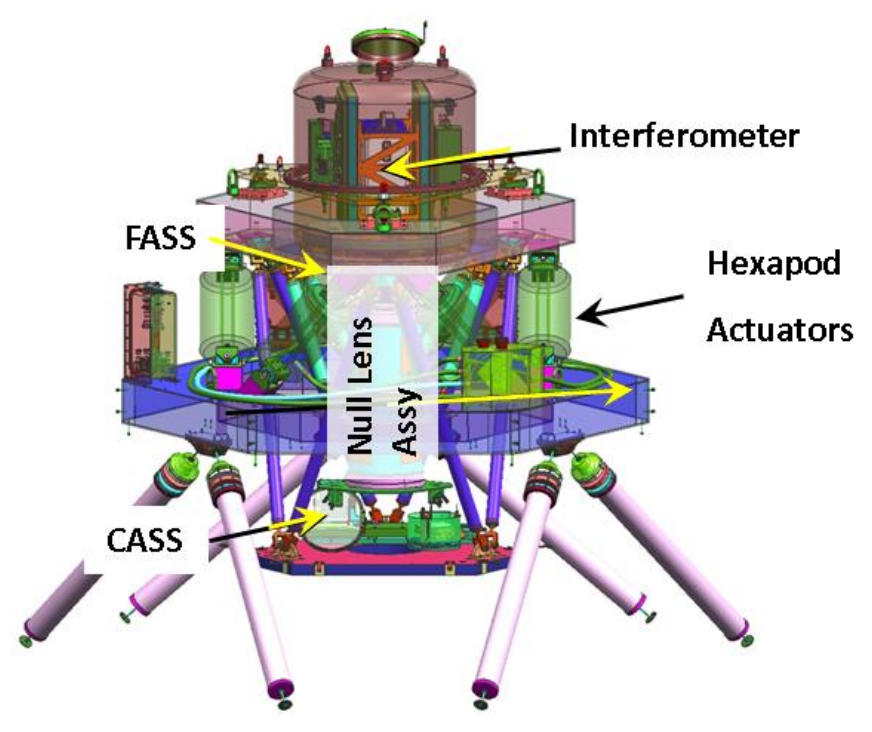

Figure 4 Schematic of inner components of COCOA located above the opening at the center of the helium shroud in the chamber.

The CASS uses the return in front of the null lens, so the light returning from the PM segments are out of focus and distorted. Nonetheless, the center of the return from both segments was easily identified, and the COCOA optical bench was moved horizontally to center the CASS field of view to the segment images (Figure 5). 


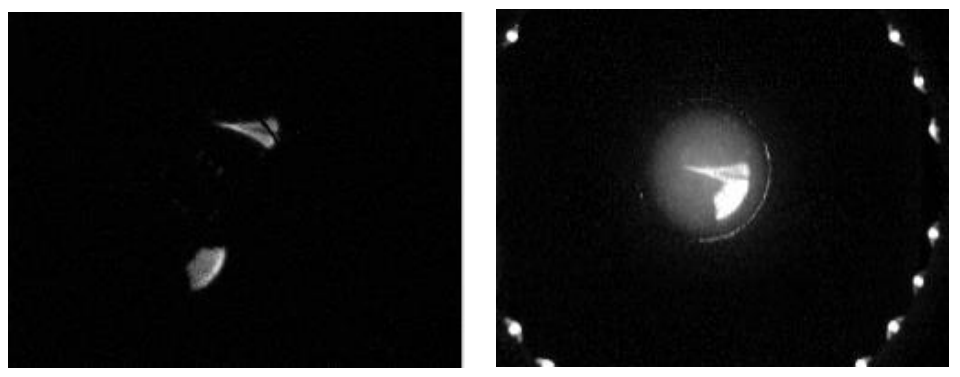

Figure 5 Centering the COCOA to the PMSA images (left) and then co-aligning the tilt of the PMSAs to the center of the field of view of the CASS (right). 

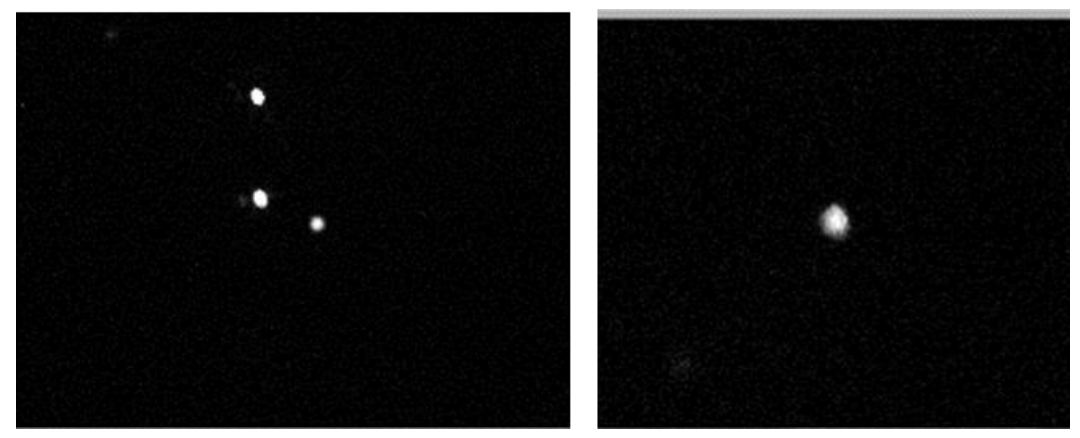

Figure 6 Light returned from the 2 mirror segments on the back side of the null lens after CASS alignment near the pinhole (left). The FASS camera image after all the light is then aligned to pass through the pinhole (right). 


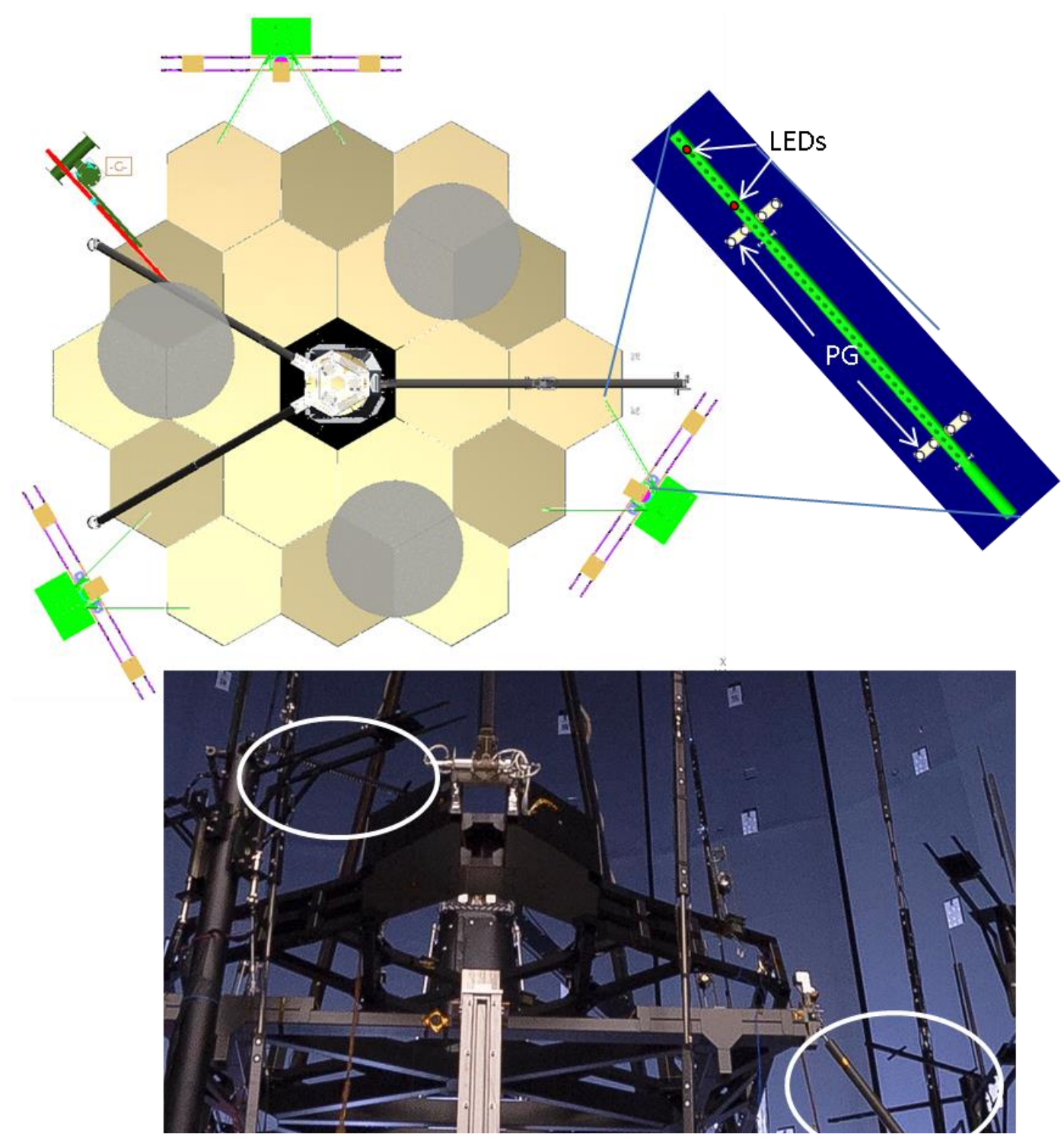

Figure 7 There are six bars over PM edge with two LEDs on the end of the bars to mark pointing direction for COCOA, 3 of the 6 bars are circled in the OGSE 2 test picture. Each bar has four PG targets. 


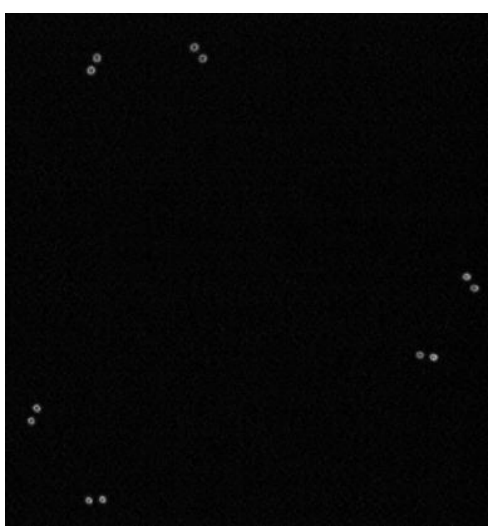

Figure 8 Image of LED fiducials in COCOA interferometer.

Once aligned into the center of the CASS, the return light will now pass through the null lens to a small hole at the top of the null lens visible by the FASS camera (Figure 6). The tilts of the PMSAs were finely adjusted to get the light inside the pinhole of the FASS.

The next step is adjusting the tilt of the COCOA. The tilt position was set using light emitting diodes located around the perimeter of the PM with PG target references indicating their position relative to the PM and AOS (Figure 7 and Figure 8).

The alignment is iteratively checked between the FASS centering and the pointing to the LED position until both alignments coincide. The pixel size in the interferometer camera corresponds to $7 \mathrm{~mm}$ at the primary mirror. With a radius of curvature of $16 \mathrm{~m}$, this limits the tilt resolution to approximately 0.4 milliradians or 1.5 arcminutes.

\section{PHASING THE PRIMARY MIRROR}

At this point, interferometric fringes appeared on the PMSAs. Normally at this point common misalignment seen between the two segments would be corrected with motion of the COCOA and uncommon misalignment between the PMSAs would be corrected with motion of the PMSAs. However, the wavefront error (WFE) from the outer uncoated segment dominated by the less polished surface figure error overly skewed and confused any common alignment calculations. So the COCOA was essentially aligned to the inner segment that was polished to flight performance levels. The radius of curvature of the inner segment was set to the nominal value determined in an earlier cryogenic test of the segment during component-level testing. The COCOA was then coarsely adjusted to the inner segment, and then the segment was finely adjusted to the interferometer. The relative alignment between the segments reached a vertical spacing position difference of $32 \mathrm{~nm}$ (Figure 9) with a repeatability of $7 \mathrm{~nm}$ (Figure 10). Note that the gap bisecting one PMSA is one of the struts holding the Secondary Mirror.

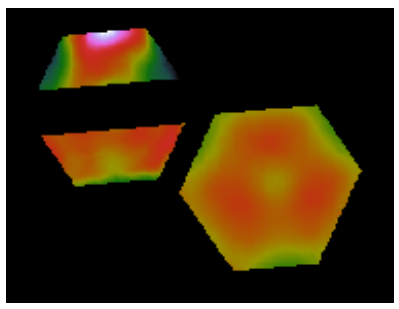

Figure 9 PMSAs aligned to a vertical (along perpendicular to optical surface) spacing of $32 \mathrm{~nm}$. 


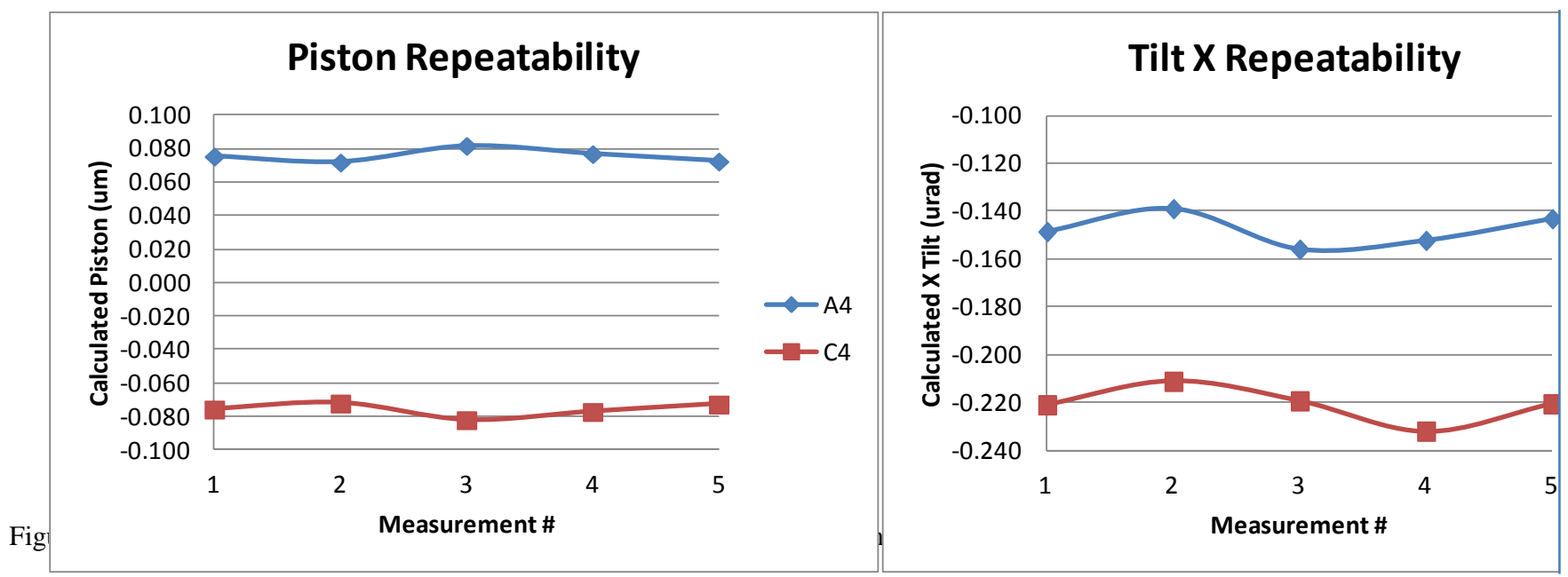

\section{SETTING THE SECONDARY MIRROR FOCUS POSITION}

After alignment, the light from the ASPA was collimated by the SMA and PMSAs to reflect from the auto-collimating flat (ACF) mirror from above to pass back through the full telescope to the detector underneath the AOS simulating a Science Instrument (Figure 1). The image can then be used to set the SM vertical position to an uncertainty of 30 micrometers.

However, the vibration of the PMSAs were higher than expected, so a new Hartmann-like technique had to be developed to analyze the images that is expected to perform nearly as well as original expectations ${ }^{6}$. Images were captured with the new technique and the analysis method is being developed to assess the images. Meanwhile, the test configuration is undergoing improvements to decrease the vibration and the vibration will be re-evaluated in an upcoming cryogenic test with the Pathfinder².

\section{DIFFERENCES WITH OTIS ALIGNMENT}

The OTIS PM will only have PG targets on the outer perimeter of mirror segments to align the phased PM relative to the AOS. The inner six segments will have no targets. However, the interferometer can measure tilt and piston differences between adjacent mirrors to tens of nanometers and nanoradians, so a contiguous primary mirror alignment is still possible with PG alignment of only the outer segment edges. After phasing the mirror, a PG measurement will check that the outer segment edges are still aligned with the AOS. Phasing and PG alignment are both iterated until the phase changes are less than the PG measurement uncertainty, if necessary.

Moreover, with PG targets spread across a much larger diameter than just the 2 segments used in the Pathfinder test and with three times the number of targets on the PM, the photogrammetry will be able to achieve tighter precision on the alignment of the PM relative to the AOS and the SM relative to the PM with the increased statistical and spatial sampling.

For the OTIS alignment a retro-reflector will be mounted on one of the PMSAs to be used with an Absolute Distance Meter to set the distance between the PM and the COCOA for setting the radius of curvature for all the PMSAs. This system was tested in the Pathfinder test with a retroreflector on a bracket rather than on one of the PMSAs.

A significant difference will be the ability to use 3 ACF mirrors around the PM aperture, the full aperture of each of the ACF mirrors, and the array of 5 Science Instruments with known relative alignments measured in previous cryogenic tests. This will increase the optical sensitivity to the alignment of the primary and secondary mirror in the image of the outward ASPA sources onto the Science Instrument detectors. 


\section{SUMMARY}

The Pathfinder test demonstrated sufficient ability to align the OTIS telescope with photogrammetry and the COCOA, and will only improve with the use of the full primary mirror size and all three planned ACF mirrors. Finer alignment of the secondary mirror with imaging from ASPA sources proved more difficult with higher than expected vibration of the PMSAs. However, the test gave valuable data to pursue improvements in the test configuration to decrease the vibration and plan alternate images to enable sufficient finer alignment of the SM in the presence of higher vibration.

\section{ACKNOWLEDGEMENTS}

The optical test design, preparation, and execution was supported by the JWST contract NNG11FD64C with NASA GSFC. The JWST system is a collaborative effort involving NASA, ESA, CSA, the Astronomy community and numerous principal investigators. The Pathfinder test and analysis were accomplished through the efforts of NASA Goddard Space Flight Center, NASA Johnson Space Center, Harris Space and Intelligence Systems, Ball Aerospace and Technologies, Genesis Engineering Solutions, and the Space Telescope Science Institute.

\section{REFERENCES}

[1] Matthews, G. W., Whitman, T. L., Feinberg, L. D., Voyton, M., Lander, J., and Keski-Kuha, R., “JWST telescope integration and test progress," Proc. SPIE 9904, (2016).

[2] Whitman, T., Knight, J. S., Waldman, M., Lightsey, P., "Non-invasive Optical End-to-End Test of a Large TMA Telescope (JWST) from the Intermediate Focus," Proc. 7436, (2009)

[3] Conrad Wells, Olczak, G., Merle, C., Dey, T., Waldman, M., Whitman, T., Wick, E., Peer, A., "The Center of Curvature Optical Assembly for the JWST Primary Mirror Cryogenic Optical Test”, Proc. SPIE 7739, (2010)

[4] Wells, C., Hadaway, J. B., Olczak, G. A., Whitman, T. L., Cosentino, J. A., Connolly, M. T., Chaney, D. M., Telfer, R. C., "Characterization of the JWST center of curvature optical assembly (CoCOA) during cryogenic testing of the JWST Pathfinder telescope," Proc. SPIE 9904, (2016)

[5] Hadaway, J. B., Wells, C., Olczak, G. A., Waldman, M., Whitman, T.L., Cosentino, J. A., Connolly, M. T., Chaney, D. M., Telfer, R. C., "Performance of the primary mirror center-of-curvature optical metrology system during cryogenic testing of the JWST Pathfinder Telescope,” Proc. SPIE 9904, (2016)

[6] Knight, J. S., Feinberg, L. D., Acton, D. S., Whitman, T. L., and Smith, K. Z., "Hartmann Test for the James Webb Space Telescope", Proc. SPIE 9904, (2016) 